\title{
Nuclear data sensitivity analysis and uncertainty propagation in the KYADJ whole-core transport code
}

\author{
$Q u \mathrm{Wu}^{1,2 *}$, Xingjie Peng ${ }^{1}$, Guanlin $\mathrm{Shi}^{2}$, Yingrui $\mathrm{Yu}^{1}$, Qing $\mathrm{Li}^{1}$, and Kan Wang ${ }^{2}$ \\ ${ }^{1}$ Science and Technology on Reactor System Design Technology Laboratory, Nuclear Power Institute of China, Chengdu, 610041, \\ China \\ ${ }^{2}$ Department of Engineering Physics, Tsinghua University, Beijing, 100084, China
}

\begin{abstract}
Nuclear data sensitivity analysis and uncertainty propagation have been extensively applied to nuclear data adjustment and uncertainty quantification in the field of nuclear engineering. Sensitivity and Uncertainty (S\&U) analysis is developed in the KYADJ whole-core transport code in order to meet the requirement of advanced reactor design. KYADJ aims to use two-dimension Method of Characteristic (MOC) and one-dimension discrete ordinate $\left(\mathrm{S}_{\mathrm{N}}\right)$ coupled method to solve the neutron transport equation and achieve onestep direct transport calculation of the reactor core. Developing sensitivity and uncertainty analysis module in KYADJ can minimize deviations caused by modeling approximation and enhance calculation efficiency. This work describes the application of the classic perturbation theory to the KYADJ transport solver. In order to obtain uncertainty, a technique is proposed for processing a covariance data file in 45-group energy grid instead of 44-group SCALE 6.1 covariance data which is extensively used in various codes. Numerical results for Uncertainty Analysis in Modelling (UAM) benchmarks and the SF96 benchmark are presented. The results agree well with the reference and the capability of S\&U analysis in KYADJ is verified.
\end{abstract}

\section{Introduction}

Nuclear data are the most important input parameters in the reactor core calculation. All nuclear data have uncertainty when they are measured by certain measuring methods and apparatus. The uncertainty information of nuclear data is stored in the evaluated nuclear data file, which can be extracted and compressed into a covariance matrix [1]. Knowledge of the uncertainty in nuclear data has an important impact on the reactor physical design. The certain confidence of the output parameter is comprehensively influenced by the uncertainty of the input parameter, the model error and the numerical error. In particular, the effects of the uncertainty of the nuclear data will domain with the improvement of computer accuracy and numerical methods.

Most developing reactor design codes strive to develop the uncertainty quantification module which is used to evaluate the effect of the input uncertainty on the output uncertainty. The evaluation approaches are generally divided into the sample-based method and the sensitivitybased method [1]. The sampling-based method is easy to use but time-consuming, especially when it is applied to rector transport codes such as Monte Carlo codes and deterministic transport codes. On the other hand, the sensitivity-based method requires massive programming but it saves time, which suits the reactor transport codes with high fidelity and accuracy.

*e-mail: q-wu15@mails.tsinghua.edu.cn
The two-dimension (2-D) Method of Characteristic (MOC) and one-dimension (1-D) discrete ordinate $\left(\mathrm{S}_{\mathrm{N}}\right)$ coupled transport code, KYADJ [2], aims to achieve the one-step direct transport calculation of the reactor core. The matched Sensitivity and Uncertainty (S\&U) analysis system is being developed in KYADJ. The paper describes a technique of applying the sensitivity-based method and verifies the sensitivity and uncertainty by the SF96 benchmark [3] and the PB-2 assembly benchmark [4].

\section{Methods}

A brief overview of the implementation of the sensitivitybased method in KYADJ and the processing of the covariance data is presented in this section.

\subsection{The Sandwich rule}

The sensitivity-based method is based on the Sandwich rule [1]:

$$
\frac{\Delta k}{k}=\mathbf{S C S}^{\mathrm{T}}
$$

where $\mathbf{S}$ is a vector composed by sensitivity coefficients, $\mathbf{C}$ is the relative covariance matrix, and $k$ represents the effective multiplication factor which is regarded as the output in this work. The sensitivity-based method contains two pivotal steps: the sensitivity calculation and the uncertainty calculation. The former obtains the sensitivity coefficients that measure how the output is sensitive to nuclear data. The latter achieves uncertainty propagation from nuclear data to the output. 


\subsection{The direct perturbation theory}

The sensitivity coefficient of $k$ to the nuclear cross section $\alpha$ is defined as

$$
S_{k, \alpha}=\frac{\alpha}{k} \frac{\partial \alpha}{\partial k}
$$

The partial derivative in Eq. (2) can be approximated by the difference in the direct perturbation theory (DPT) [5]:

$$
S_{k, \alpha}=\frac{\alpha}{k} \frac{\partial \alpha}{\partial k} \approx \frac{\alpha}{k} \frac{k(\alpha+\delta \alpha)-k(\alpha)}{\delta \alpha}
$$

When $\alpha$ is perturbed slightly, a slight change occurs on $k$. The approximation becomes more and more accurate when the perturbation of $\alpha$ is as small as possible without being overwhelmed by truncation errors. The disadvantage of DNP is time-consuming. Nevertheless, it can be used as a reference method as its implementation does not require programming.

\subsection{The classical perturbation theory}

In general, $k$ is solved by the $k$-eigenvalue neutron transport equation which is can be written into

$$
\mathbf{A} \Phi=\frac{1}{k} \mathbf{B} \Phi
$$

where $\boldsymbol{\Phi}$ is the neutron flux, Aand $\mathbf{B}$ are the neutron loss operator and the production operator. The sensitivity coefficient can be derived from the classical perturbation theory (CPT) [6] which is expressed as

$$
S_{k, \alpha}=-\frac{\alpha}{k} \frac{\left\langle\left(\frac{\partial \mathbf{A}}{\partial \alpha}-\frac{1}{k} \frac{\partial \mathbf{B}}{\partial \alpha}\right) \boldsymbol{\Phi}, \boldsymbol{\Phi}^{*}\right\rangle}{\frac{1}{k^{2}}\left\langle\mathbf{B} \boldsymbol{\Phi}, \boldsymbol{\Phi}^{*}\right\rangle}
$$

where $\boldsymbol{\Phi}^{*}$ is the adjoint flux which is solved by the adjoint form of Eq. (4).

It is crucial to solve the neutron flux and the adjoint flux accurately. For a 3-D reactor core, the 2-D MOC and 1-D $S_{N}$ coupled method is used to solve the neutron transport equation and adjoint neutron transport equation and provides the fine pin-by-pin flux and adjoint flux distribution. The iteration process is accelerated by the 3-D CMFD [2].

\subsection{The processing of the covariance data}

The multigroup (relative) covariance data are processed with the NJOY [7] code from ENDF/B-VII.1 and conform to the 45-group structure of the multigroup nuclear data library employed by KYADJ. The multigroup nuclear data library contains the total capture and scattering cross section other than the individual capture and scattering cross section. The covariance data for the total capture and scattering cross section are reprocessed by the Sandwich rule as the total capture and scattering cross section are the linear sums of the individual capture and scattering cross section [8].

\section{Results}

Application cases contain the SF96 benchmark from the Takahama-3 PWR and the PB-2 assembly benchmark from the Benchmarks for Uncertainty Analysis in Modeling (UAM). The sensitivity coefficients are verified by DPT and the uncertainty quantification is performed for both cases. It is noted that the sensitivity analysis function and the covariance matrix has been verified in comparison with the Reactor Monte Carlo Code (RMC) in previous study[9].

\subsection{The SF96 benchmark}

The SF96 benchmark model is presented in Fig. 1. The radial high heterogeneity is implemented to test the 2-D MOC solution. The group-dependent sensitivity coefficients for all types of the cross section of all nuclides are analyzed and compared with those calculated by DNP. The automatic execution scripts for DNP are implemented in KYADJ to calculate the sensitivity coefficients by perturbing the multigroup cross section seriatim.

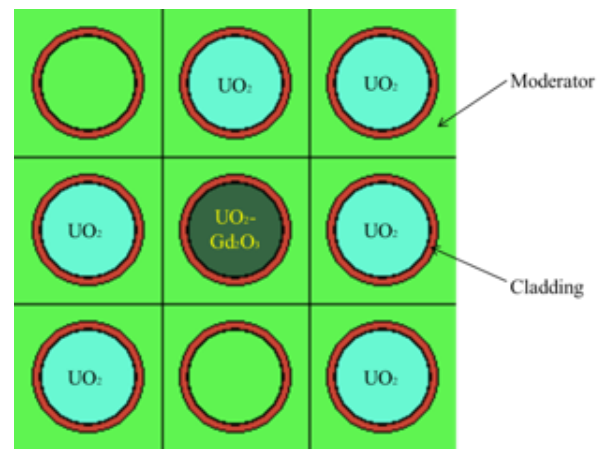

Figure 1. The SF96 benchmark model

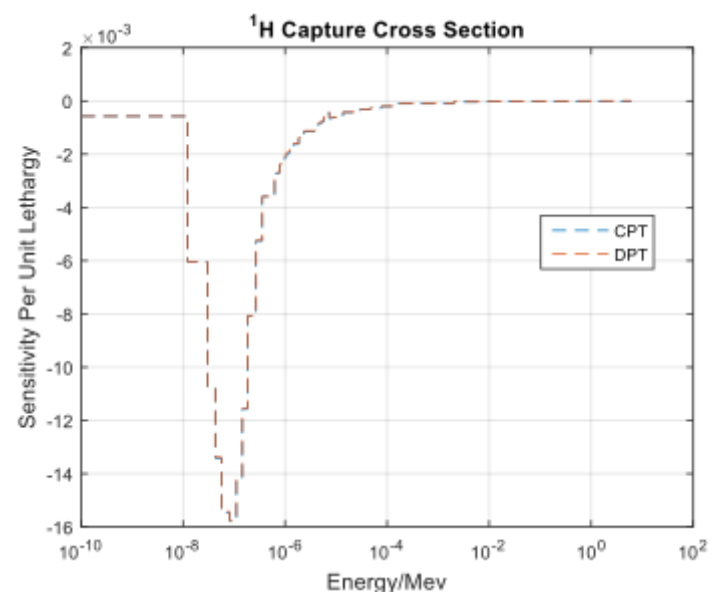

Figure 2. The sensitivity per unit lethargy of $k$ to the ${ }^{1} \mathrm{H}$ capture cross sections

The sensitivity per unit lethargy of $k$ to the ${ }^{1} \mathrm{H}$ capture cross section, the ${ }^{1} \mathrm{H}$ scatting cross section, and the ${ }^{238} \mathrm{U}$ capture cross section are shown in Fig. 2 Fig. 4, respectively. The accordance between CPT and DNP is achieved 


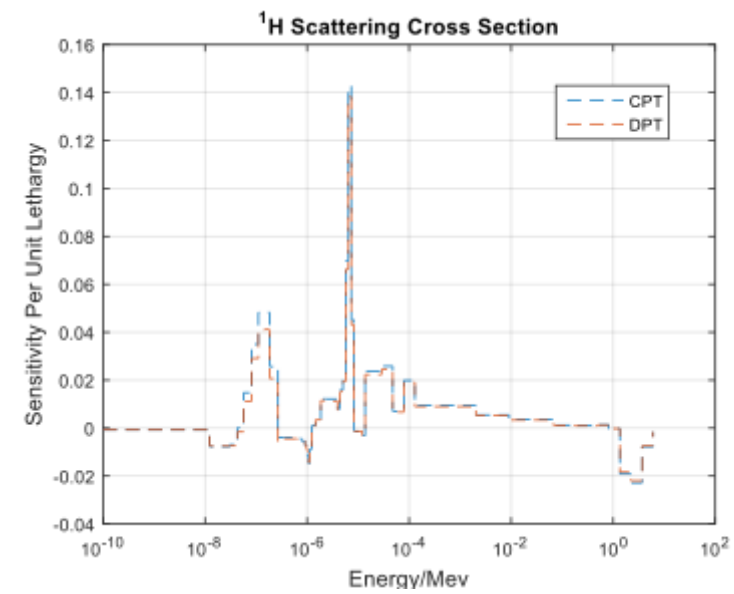

Figure 3. The sensitivity per unit lethargy of $k$ to the ${ }^{1} \mathrm{H}$ scattering cross sections

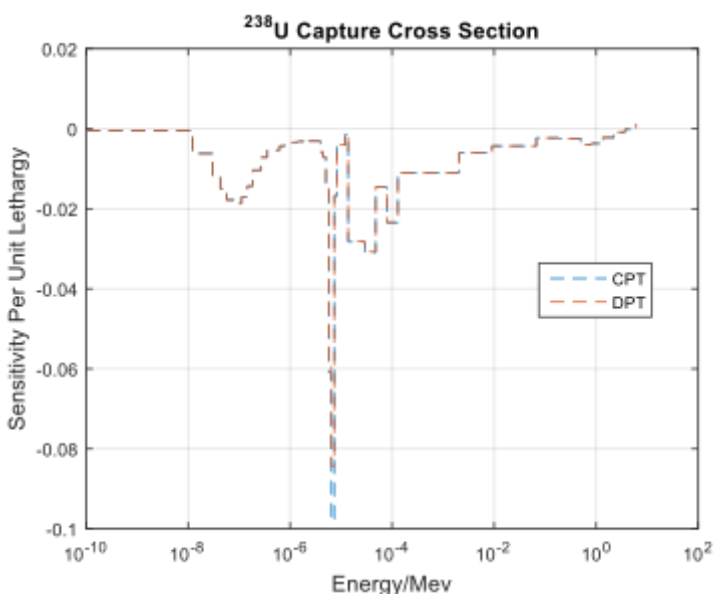

Figure 4. The sensitivity per unit lethargy of $k$ to the ${ }^{238} U$ capture cross sections

in the verification, which implies that KYADJ has the ability to perform the sensitivity analysis.

The eight most significant total sensitivity coefficients of $k$ to the cross section and the corresponding uncertainty information are listed in Table $1 . k$ is the most sensitive to the ${ }^{235} \mathrm{U}$ average fission neutron number. Besides, some cross section such as the ${ }^{1} \mathrm{H}$ scattering cross section with the large total sensitivity coefficient leads to a small contribution to the uncertainty $\frac{\Delta k}{k}$.

\subsection{The PB-2 assembly benchmark}

The PB-2 assembly benchmark model is shown in Fig. 5. The axial is divided into three MOC planers according to the configuration of the rod 5A. The two main nuclides, ${ }^{235} \mathrm{U}$ and ${ }^{238} \mathrm{U}$, are analyzed in this section.

The sensitivity coefficients of $k$ with respect to the ${ }^{235} \mathrm{U}$ and ${ }^{238} \mathrm{U}$ scattering and capture cross sections are plotted in Fig. 6. Table 3.2 shows the uncertainty sources from the ${ }^{235} \mathrm{U}$ and ${ }^{238} \mathrm{U}$ cross sections together with the total sensitivity coefficients. The total uncertainty contribution to $\frac{\Delta k}{k}$ of ${ }^{238} \mathrm{U}$ is larger than that of ${ }^{235} \mathrm{U}$.
Table 1. The eight most significant total sensitivity coefficients of $k$ to the cross section and the corresponding uncertainty

\begin{tabular}{lllll}
\hline Nuclide & ND & CPT & DPT & $\frac{\Delta k}{k}(\%)$ \\
\hline${ }^{235} \mathrm{U}$ & $\bar{v}$ & $9.30 \mathrm{E}-01$ & $9.30 \mathrm{E}-01$ & $1.23 \mathrm{E}-01$ \\
${ }^{235} \mathrm{U}$ & $\sigma_{f}$ & $4.80 \mathrm{E}-01$ & $4.73 \mathrm{E}-01$ & $1.52 \mathrm{E}-01$ \\
${ }^{238} \mathrm{U}$ & $\sigma_{c}$ & $-1.91 \mathrm{E}-01$ & $-1.89 \mathrm{E}-01$ & $2.23 \mathrm{E}-01$ \\
${ }^{1} \mathrm{H}$ & $\sigma_{s}$ & $1.38 \mathrm{E}-01$ & $1.22 \mathrm{E}-01$ & $5.97 \mathrm{E}-03$ \\
${ }^{235} \mathrm{U}$ & $\sigma_{c}$ & $-9.87 \mathrm{E}-02$ & $-9.90 \mathrm{E}-02$ & $1.23 \mathrm{E}-01$ \\
${ }^{238} \mathrm{U}$ & $\bar{v}$ & $7.00 \mathrm{E}-02$ & $7.00 \mathrm{E}-02$ & $8.11 \mathrm{E}-02$ \\
${ }^{157} \mathrm{Gd}$ & $\sigma_{f}$ & $-5.09 \mathrm{E}-02$ & $-4.62 \mathrm{E}-02$ & $2.20 \mathrm{E}-01$ \\
${ }^{238} \mathrm{U}$ & $\sigma_{f}$ & $4.54 \mathrm{E}-02$ & $4.54 \mathrm{E}-02$ & $2.35 \mathrm{E}-02$ \\
\hline
\end{tabular}

\begin{tabular}{|c|c|c|c|c|c|c|}
\hline 4 & 3 & 3 & 2 & 2 & 2 & 3 \\
\hline 3 & 2 & 1 & 1 & 1 & 1 & 2 \\
\hline 3 & 1 & $5 \mathrm{~A}$ & 1 & 1 & $5 \mathrm{~A}$ & 1 \\
\hline 2 & 1 & 1 & 1 & 1 & 1 & 1 \\
\hline 2 & 1 & 1 & 1 & $6 \mathrm{~B}$ & 1 & 1 \\
\hline 2 & 1 & $5 \mathrm{~A}$ & 1 & 1 & 1 & 2 \\
\hline 3 & 2 & 1 & 1 & 1 & 2 & 2 \\
\hline
\end{tabular}

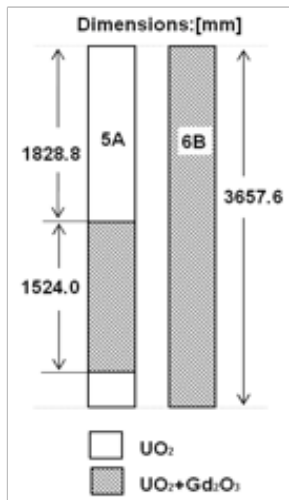

Figure 5. The PB-2 assembly benchmark model

Table 2. The uncertainty of $k$ caused by the ${ }^{235} \mathrm{U}$ and ${ }^{238} \mathrm{U}$ cross sections for the PB-2 assembly benchmark

\begin{tabular}{llll}
\hline Nuclide & ND & CPT & $\frac{\Delta k}{k}(\%)$ \\
\hline${ }^{235} \mathrm{U}$ & $\sigma_{c}, \sigma_{c}$ & $-1.15 \mathrm{E}-01$ & $1.44 \mathrm{E}-01$ \\
${ }^{235} \mathrm{U}$ & $\bar{v}, \bar{v}$ & $8.82 \mathrm{E}-01$ & $1.15 \mathrm{E}-01$ \\
${ }^{235} \mathrm{U}$ & $\sigma_{f}, \sigma_{f}$ & $3.78 \mathrm{E}-01$ & $1.14 \mathrm{E}-01$ \\
${ }^{235} \mathrm{U}$ & $\sigma_{f}, \sigma_{c}$ & - & $7.59 \mathrm{E}-02$ \\
${ }^{235} \mathrm{U}$ & $\sigma_{s}, \sigma_{c}$ & - & $2.68 \mathrm{E}-02$ \\
${ }^{235} \mathrm{U}$ & $\sigma_{s}, \sigma_{s}$ & $9.37 \mathrm{E}-03$ & $2.52 \mathrm{E}-02$ \\
& & Total & $5.01 \mathrm{E}-01$ \\
${ }^{238} \mathrm{U}$ & $\sigma_{c}, \sigma_{c}$ & $-3.51 \mathrm{E}-01$ & $4.21 \mathrm{E}-01$ \\
${ }^{238} \mathrm{U}$ & $\sigma_{s}, \sigma_{s}$ & $-1.11 \mathrm{E}-02$ & $2.18 \mathrm{E}-01$ \\
${ }^{238} \mathrm{U}$ & $\bar{v}, \bar{v}$ & $1.18 \mathrm{E}-01$ & $1.37 \mathrm{E}-01$ \\
${ }^{238} \mathrm{U}$ & $\sigma_{f}, \sigma_{f}$ & $7.08 \mathrm{E}-02$ & $3.66 \mathrm{E}-02$ \\
${ }^{238} \mathrm{U}$ & $\sigma_{s}, \sigma_{c}$ & - & $2.62 \mathrm{E}-02$ \\
${ }^{238} \mathrm{U}$ & $\sigma_{s}, \sigma_{f}$ & - & $7.61 \mathrm{E}-03$ \\
${ }^{238} \mathrm{U}$ & $\sigma_{f}, \sigma_{c}$ & - & $1.11 \mathrm{E}-03$ \\
& & Total & $8.48 \mathrm{E}-01$ \\
\hline
\end{tabular}




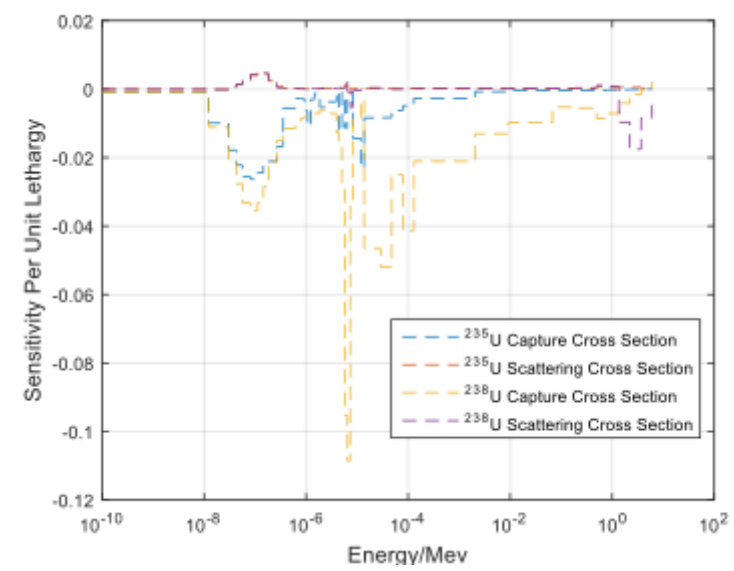

Figure 6. The sensitivity per unit lethargy of $k$ to the ${ }^{235} \mathrm{U}$ and ${ }^{238} \mathrm{U}$ capture and scattering cross sections

\section{Summary}

The sensitivity-based method is successfully implemented in the 2-D/1-D coupled transport code KYADJ. The 3-D neutron transport equation and adjoint transport equation are solved and the fine neutron flux and adjoint flux distributions are used to perform the calculation of the sensitivity coefficients of the multiplication factor based on CPT. A series of verification calculations are performed, using the SF96 benchmark and the PB-2 assembly benchmark. The accordance between CPT and DPT has been achieved and the conclusion that KYADJ has the ability to perform the sensitivity and uncertainty analysis can be arrived. Future work devotes to develop the sensitivity and uncertainty analysis competence for other responses such as reaction rates and the fission power. The generalized perturbation theory (GPT) will be used and the 3-D generalized fix-source equation will be solved in the framework of KYADJ.

\section{References}

[1] D.G. Cacuci, Sensitivity and Uncertainty Analysis, vol. 1, Chapman \& Hall/CRC, Boca Raton, Fla, USA, 2003

[2] Q. Wu, et al., "Whole-core Forward-adjoint Neutron Transport Solutions with Coupled 2-D MOC and 1-D $\mathrm{S}_{\mathrm{N}}$ and Kinetics Parameter Calculation," , Prog. Nucl. Energy 108, 310-318 (2018)

[3] H.M. Dalle, "Monte Carlp Burnup Simulation of the Takahama-3 Benchmark Experiment," 2009 International Nuclear Atlantic Conference - INAC 2009, Rio de Janeiro, RJ, Brazil, September 27-October 2 (2009)

[4] K. Ivanov, et al., "Benchmarks for Uncertainty Analysis in Modelling (UAM) for the Design, Operation and Safety Analysis of LWRs. Volume I," NEA/NSC/DOC(2013), available from OECD/NEA Data Bank

[5] Y. Liu, et al., "Implicit Sensitivity and Uncertainty Analysis with the Subgroup Resonance Calculation Method," Ann. Nucl. Energy 79, 18-26 (2015)

[6] R.L. Childs, "Generalized Perturbation Theory Using Two Dimensional Discrete Ordinates Transport Theory," Tech. Rep. ORNL/CSD/TM-127, Oak Ridge National Laboratory, Oak Ridge, Tenn, USA (1980)

[7] D.W. Muir, R.M. Boicourt, A.C. Kahler, "The NJOY Nuclear Data Processing System, Version 2012," LAUR-12-27079 (2012)

[8] M. Pusa, "Incorporating Sensitivity and Uncertainty Analysis to a Lattice Physics Code with Application to CASMO-4," Ann. Nucl. Energy 40, 153-162 (2012)

[9] Q. Wu, et al., "Eigenvalue sensitivity and uncertainty analysis based on a 2-D/1-D whole-core transport code KYADJ,” Ann. Nucl. Energy 122, 185-192 (2018) 